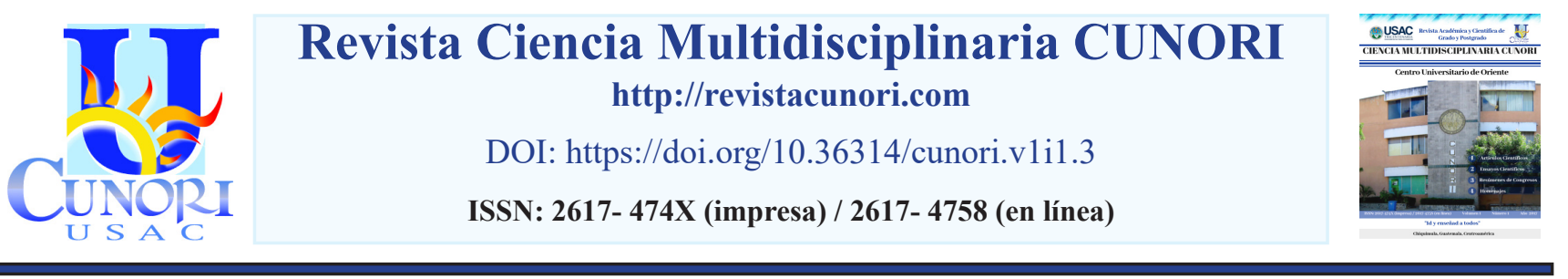

Como citar el artículo

Tax J., Mazariegos E. (2017). Relación de la onda Q y la obesidad. Revista Ciencia Multidisciplinaria CUNORI, 1(1), 1-6. DOI: https://doi.org/10.36314/cunori.v1i1.3

\title{
Relación de la onda Q y la obesidad
}

\section{Obesity relation of the wave Q}

\author{
Josué Tax*, Edvin Mazariegos \\ Centro Universitario de Oriente (CUNORI), Universidad de San Carlos de Guatemala \\ Recibido: 08 de marzo de 2017 / Aceptado: 18 de junio de 2017 \\ Disponible en internet el 30 de noviembre de 2017 \\ *Autor para correspondencia. \\ Correo electrónico: taxmonge@yahoo.es
}

Resumen

$\mathrm{L}$ a obesidad es un síndrome que se caracteriza por el aumento de la masa corporal, consecuencia de la mala nutrición, constituyendo un riesgo para la salud. Se asocia con alteraciones electrocardiográficas tempranas sin síntomas clínicos, se plantea que una de las causas de la prolongación de la onda Q en DIII es el aumento de la grasa corporal. Se realizó el estudio Analítico Comparativo sobre la relación de la Onda Q y la obesidad en 50 pacientes obesos y 50 no obesos en una muestra al azar de quienes acudieron a consulta externa del Hospital "Carlos Manuel Arana Osorio" Chiquimula, en marzo-mayo de 2015. Se realizó Electrocardiograma (ECG) a los pacientes estando en reposo y los que presentaron onda Q, se hizo ECG control estando el paciente en inspiración profunda y sostenida por 10 segundos. Se determinó, que los pacientes obesos tienen un riesgo 17 veces mayor de presentar onda Q patológica en DIII comparado con los no obesos, con una relación estadísticamente significativa $(\mathrm{OR}=17.5$ valor $\mathrm{p}=0.00$ ), la edad promedio mayoritaria fue en el intervalo de 45-51 años, con predominio en el sexo femenino con una relación 2:1. A la inspiración profunda y sostenida, la Onda Q en DIII desaparece o disminuye de voltaje en el $34 \%$ de los pacientes.

Palabras clave: obesidad, electrocardiograma, índice de masa corporal, onda Q en DIII, ventrículo izquierdo

Abstract

$\mathrm{O}^{\mathrm{b}}$ besity is a syndrome characterized by an increase in body mass as a consequence of poor nutrition, constituting a risk to health. It is associated with early electrocardiographic changes without clinical symptoms, it is suggested that one of the causes of the prolongation of the Q wave in DIII is the increase in body fat. The Comparative Analytical study was carried out on the relation of Wave Q and obesity in 50 obese patients and 50 non-obese in a random sample of those who attended the outpatient clinic of the Hospital "Carlos Manuel Arana Osorio" Chiquimula, in March-May 2015. Electrocardiogram (ECG) was performed on patients who were at rest and those who presented with a Q wave. ECG was done while the patient was in deep and sustained inspiration for 10 seconds. It was determined that obese patients have a 17 times higher risk of presenting pathological Q wave in DIII compared with non-obese patients, with a statistically significant relationship $(\mathrm{OR}=17.5 \mathrm{p}$ value $=0.00)$, the average age was in the range of 45-51 years, with predominance in the female sex with a 2: 1 ratio. At deep and sustained inspiration, wave Q in DIII disappears or decreases in voltage in 34\% of patients.

Key words: obesity, electrocardiogram, body mass index, wave Q in DIII, left ventricle 


\section{Introducción}

El electrocardiograma es la representación gráfica de la actividad eléctrica del corazón que se obtiene mediante un electrocardiógrafo en forma de cinta continua. Es el instrumento principal de la electrofisiología cardiaca y tiene una función relevante en el cribado y diagnóstico de las enfermedades cardiovasculares, alteraciones metabólicas y a la predisposición a una muerte súbita cardiaca (Dilaveris et al 2000). La obesidad mórbida es una patología que presenta un aumento espectacular en los últimos años, situación que se llega a denominar la "epidemia del siglo XXI". Las consecuencias, tanto para el individuo y como para la sociedad es importante, ya que la obesidad provoca complicaciones para la salud tanto metabólicas como diabetes, dislipidemia, hipertensión arterial, problemas relacionados con el sueño (SAOS), como dificultades mecánicas como artritis que son factores de riesgo muy bien identificados como causa de mortalidad en este tipo de pacientes (Guzmán et al 2001).

La terapia médica que busca disminuir el exceso de peso en pacientes obesos se basa en el tratamiento endocrinológico especializado con modificaciones del comportamiento, dietarias y de actividad física. Este tipo de tratamiento ha mostrado pobres resultados a largo plazo ya que los pacientes vuelven a ganar peso en los años siguientes al tratamiento (Seyfeli et al 2006).

En el caso de la obesidad mórbida, cuando han fracasado los tratamientos convencionales (dietas, medicamentos y psicoterapia), hay que tomar en cuenta la técnica de electrocardiograma, porque en un elevado número de pacientes se normalizan las cifras de Onda QIII, sin necesidad de medicamentos, al igual que mejoran otras complicaciones. La Electrocardiografía constituye una alternativa terapéutica eficaz en estos pacientes, debido a la aparición silenciosa de patologías cardiacas diversas, por lo que es de importancia contar con un equipo interdisciplinario entrenado en el manejo electrocardiográfico.

La preparación y el seguimiento médico-nutricional del paciente son decisivos para el éxito (Chávez, 2013). Onda Q prominente (patológica): Mayor a $0.04 \mathrm{~ms}$ y $/$ o mayor a $0.1 \mathrm{mV}$, aquella cuya profundidad es mayor a un cuarto de la altura de la onda $\mathrm{R}$ que le sigue; consideraríamos patológica según los criterios de voltaje. Al momento de tomar el ECG notamos que en el paciente es obeso, la onda Q está presente en DIII, no hay en DII y/o aVF, como debería ser si hablamos de un infarto de miocardio inferior (González 2008).

\section{Materiales y métodos}

\section{Fuente de información y registro de datos}

Se realizó un estudio analítico en 50 pacientes obesos y 50 no obesos con similares características tomando una muestra al azar, de quienes asistieron a consulta externa del Hospital Nacional "Carlos Manuel Arana Osorio" Chiquimula.

Fueron incluidos aquellos pacientes que presentaron un IMC normal $(18-25 \mathrm{~kg} / \mathrm{m} 2)$ y pacientes que presentaron algún grado de obesidad (IMC $\geq 26 \mathrm{~kg} / \mathrm{m} 2$ ), todos atendidos en los meses de marzo - mayo de 2015. Se utilizó una confiabilidad del $95 \%$, y se trabajó con una muestra de 100 pacientes. Se estudiaron las variables edad, sexo, obesidad, onda Q en DIII. 
Se solicitó autorización a las autoridades superiores correspondientes para realizar Electrocardiograma a pacientes que presenten Índice de Masa Corporal (IMC) normal y obesos. Posteriormente se analizó cada Electrocardiograma y el paciente que presentó Onda Q en DIII patológica, se le realizó un ECG control, con el paciente estando en inspiración profunda y sostenida por 10 segundos, se hizo comparación de ECG, para determinar cuál es la relación y frecuencia de la Onda Q en DIII en personas obesas y no obesas, y determinar si la onda $Q$ en DIII desaparece o disminuye de voltaje estando el paciente en inspiración profunda y sostenida.

\section{Resultados}

De 100 pacientes (50 obesos y 50 con IMC normal), 35 pacientes con IMC $\geq 30 \mathrm{~kg} / \mathrm{m} 2$ que equivalen a un $70 \%$, presentaron onda Q patológica, 2 pacientes con IMC entre $20-29 \mathrm{~kg} / \mathrm{m} 2$ que equivale un $4 \%$, mostraron onda $\mathrm{Q}$ patológica.

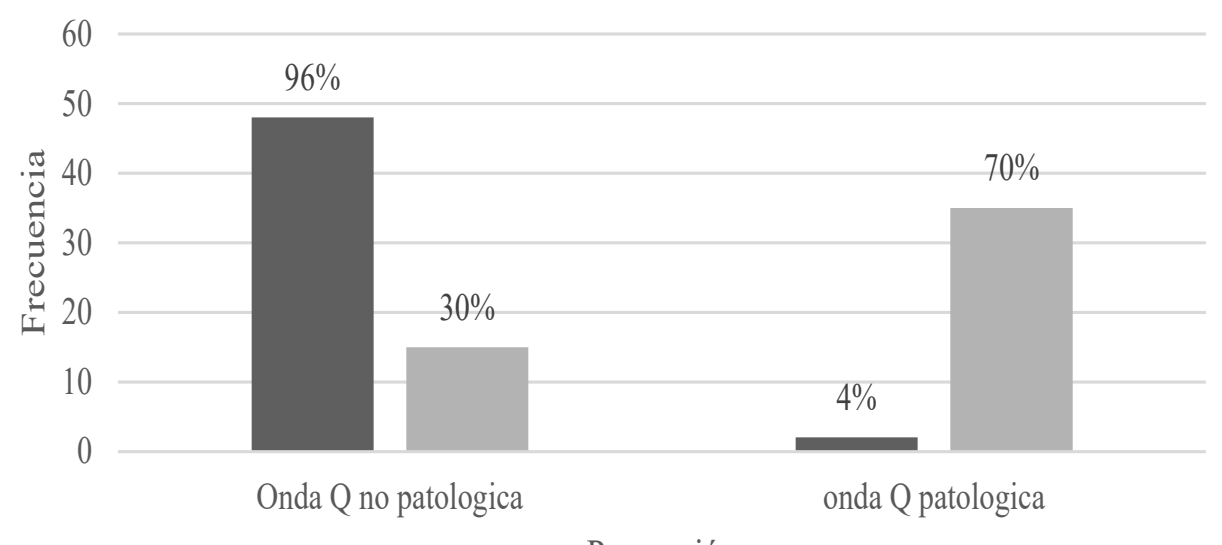

Proporción

- IMC $20-29 \mathrm{~kg} / \mathrm{m} 2 \quad$ IMC $\geq 30 \mathrm{~kg} / \mathrm{m} 2$

Figura 1. Proporción de pacientes con onda Q patológica en relación a su IMC en pacientes de la consulta externa del Hospital Nacional "Carlos Manuel Arana Osorio" entre marzo y mayo de 2015.

Fueron analizadas la relación de onda Q en DIII del ECG según el sexo, siendo el sexo femenino con mayor predominio con una relación 2:1. La edad promedio de pacientes con presencia de onda $\mathrm{Q}$ se encuentra entre 45-51 años. 
Tabla 1. Relación entre onda Q y obesidad en pacientes de la consulta externa del Hospital Nacional "Carlos Manuel Arana Osorio" entre marzo y mayo de 2015.

\begin{tabular}{|l|c|c|}
\hline & $\begin{array}{l}\text { Onda Q en } \\
\text { DIII }\end{array}$ & $\begin{array}{l}\text { Onda Q no } \\
\text { presente }\end{array}$ \\
\hline Obesos & 35 & 15 \\
\hline No obesos & 2 & 48 \\
\hline & $\begin{array}{l}\text { Total:100 } \\
\text { pacientes }\end{array}$ & \\
\hline
\end{tabular}

Existe una relación entre la aparición de onda Q y la Obesidad. Los Obesos tienen 17 veces más riesgo de presentar onda Q en DIII (tabla 1) al compararlos con pacientes con índice de masa corporal normal (OR: 17.5 con un valor $\mathrm{P}$ de 0.0000$)$.

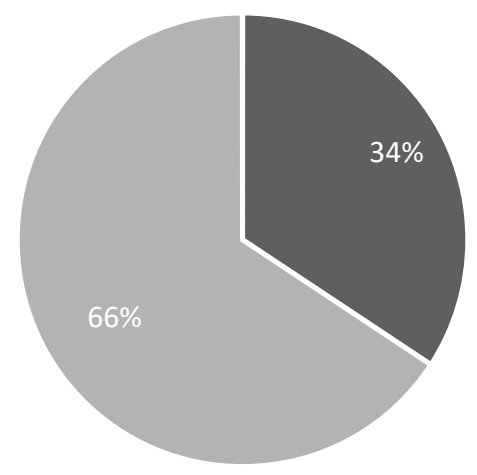

- Desaparición de onda Q o disminución del voltaje en inspiración = onda Q en DIII

Figura 2. Cambios de onda Q en DIII durante inspiración en pacientes de la consulta externa del Hospital Nacional "Carlos Manuel Arana Osorio" entre marzo y mayo de 2015.

A los pacientes que presentaron onda Q en DIII, se les realizó un ECG control en inspiración profunda y sostenida durante 10 segundos de los cuales en un 34\% (12) desapareció la onda Q en DIII o disminuyó su voltaje.

\section{Discusión}

La obesidad se asocia a múltiples patologías, principalmente de origen cardiovascular (Rippe et al 1998). En este estudio observamos varios hallazgos: Los pacientes obesos, tienen un riesgo 17 veces mayor de presentar onda Q patológica en DIII, comparado con los no obesos, con una relación estadísticamente significativa, por lo cual es importante tratar la obesidad para prevenir trastornos de la función cardiaca. Estos datos no se asociaron a otros factores como edad, tabaquismo o DM. 
Es bien sabido que los pacientes con obesidad están bajo riesgo de arritmias ventriculares y muerte súbita, además actualmente hay estudios donde se asocia la obesidad a riesgo de FA en un 50\%. El estudio Framingham concluyó que existe un incremento en la mortalidad en sujetos con un índece de masa corporal mayor de $2727 \mathrm{~kg} / \mathrm{m} 2$, siendo éste uno de los mayores factores de riesgo coronario (Mclnnis et al 1999). A la inspiración profunda y sostenida por 10 segundos, la onda Q en DIII, desaparece o disminuye de voltaje en el 34\% de los pacientes, lo que demuestra que puede ser posicional la aparición de onda Q y no indicamos patología cardíaca.

\section{Agradecimientos}

Al Coordinador de la carrera de Médico y Cirujano Dr. Edvin Danilo Mazariegos Albanés por el apoyo incondicional y asesorar la investigación, al personal del Hospital Nacional "Carlos Manuel Arana Osorio" por su apoyo para desarrollar este y otros proyectos, así como su entrega profesional al servicio de los pacientes, al Ingeniero Christian Sosa Sancé por su colaboración en estadística, a la Dirección General de Investigación de la Universidad de San Carlos de Guatemala, por permitir participar en revistas científicas.

\section{Referencias bibliográficas}

Chávez, E. 2015. Dispersión de la onda P en obesos, un factor de riesgo para la Fibrilación Atrial. Revista Iberoamericana de Arritmología 4(1):136-139. https://doi.org/10.5031/v4i1.RIA10228

Chávez, E; Hevia, J; Rodríguez, E. 2010. Dispersión de la Onda P en el Electrocardiograma con Bases en el Potencial de Acción Auricular y en la Heterogeneidad del impulso en Aurículas. Relampa 23(3): 126-133.

Dilaveris, E; Gialafos, E; Andrikopoulos, G; Ritcher, D; Papanikolaou, V; Polaris, K; Gialafos, J. (2006). Clinical and electrocardiographic predictors of recurrent atrial fibrillation (en línea). Pacing Clin Electrophysiol. 30(6):957-961. https://doi.org/10.1111/j.1540-8159.2000.tb06761.x

González, J. 2008. La onda Q profunda en la III derivación del ECG. Importancia de sus variaciones durante la inspiración profunda. Cátedra de Clínica Médica del Prof. J. J. Spangenberg, y Dispensario N" 5 de Asistencia al Cardiaco.

Guzmán, A; Sánchez, O; Montez, M; Diaz, S; Vásquez, J; Pérez, M. 2001.Capacidad cardiovascular en pacientes obesos. Revista Mexicana de Medicina Física y Rehabilitación 13(4):109-112.

Mclnnis, K; Bader, D; Pierce, G; Balady,G. 1999. Comparison of cardiopulmonary responses in obese women using ramp versus step treadmill protocols. The American Journal of Cardiology 83(2):289291. https://doi.org/10.1016/S0002-9149(98)00843-1

Rippe, J; Crossley, S, Ringer, R. 1998. Obesity as a chronic disease; modern medical and lifestyle management. J Am Diet Assoc 9-15. https://doi.org/10.1016/S0002-8223(98)00704-4

Seyfeli, E; Duru, M; Kuvandiik, G.2006. Effect of obesity on P wave dispersión and QT dispersión in Obese subjects. The Anatiolian journal of cardiology 6(2):126-129. https://doi.org/10.1038/sj.ijo.0803233 


\section{Sobre el autor}

\section{Josué Tax}

Estudiante de la licenciatura en la carrera Médico y Cirujano del Centro Universitario de Oriente CUNORI, de la Universidad de San Carlos de Guatemala. Su línea de investigación es sobre cardiología y medicina interna.

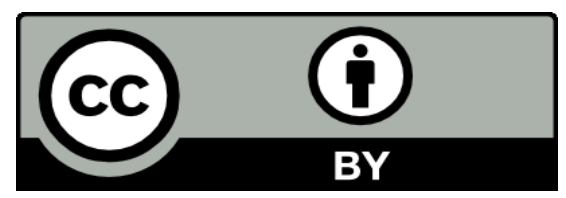

Este texto está protegido por una licencia CreativeCommons 4.0.

Usted es libre para compartir, copiar y redistribuir el material en cualquier medio o formato y adaptar el documento, remezclar, transformar y crear a partir del material para cualquier propósito, incluso comercialmente, siempre que cumpla la condición de atribución: usted debe reconocer el crédito de una obra de manera adecuada, proporcionar un enlace a la licencia, e indicar si se han realizado cambios. Puede hacerlo en cualquier forma razonable, pero no de forma tal que sugiera que tiene el apoyo del licenciante o lo recibe por el uso que hace. 